\title{
PERMANENT GENETIC RESOURCES Isolation and characterization of microsatellite markers for Carolina hemlock (Tsuga caroliniana)
}

\author{
S. A. JOSSERAND,* K. M. POTTER,+C. S. ECHT* and C. D. NELSON* \\ *US Department of Agriculture Forest Service, Southern Research Station, Southern Institute of Forest Genetics, 23332 Mississippi 67, \\ Saucier, MS 39574, USA, +North Carolina State University, 3041 Cornwallis Road, Research Triangle Park, NC 27709, USA
}

\begin{abstract}
We describe the isolation and characterization of 31 polymorphic di- and trinucleotide microsatellite marker loci for Carolina hemlock (Tsuga caroliniana Englem.). In addition, primer pairs for 16 loci amplified scoreable alleles in six other Tsuga species. In eastern North America, both Carolina hemlock and eastern hemlock (Tsuga canadensis [L.] Carr.) populations are declining due to infestation by hemlock woolly adelgid, Adelges tsugae. The markers described here should enhance population genetic studies of hemlocks, providing valuable information for conserving and restoring these important forest tree species.
\end{abstract}

Keywords: conservation genetics, hemlock, SSR markers

Received 5 March 2008; revision accepted 15 May 2008

Carolina hemlock is endemic to the southern Appalachian Mountains of eastern North America. It is a wind-pollinated species with a restricted range confined to isolated rocky slopes and ridges (Farjon 1990). In recent years, this rare and vulnerable species, which has a G3 global conservation priority rank (www.natureserve.org/explorer/), has begun to experience mortality due to infestation by the hemlock woolly adelgid (HWA), Adelges tsugae Annand, an exotic insect that has caused a rapid decline of eastern hemlock across much of its range (McClure et al. 2003).

Microsatellite markers were developed from fresh leaf tissue DNA collected from a single Tsuga caroliniana tree (tree \#247, Linville Falls, North Carolina). Enrichment, cloning and sequencing were performed (Genetic Identification Services) as previously described (Josserand et al. 2006), with the exception that the clone libraries were enriched for AC, GA and ATG simple sequence repeats (SSR). From each library, 53 recombinant colonies were selected at random and their cloned fragments were sequenced. Relatively long SSRs that were centrally located in the sequence were identified for 135 of these inserts: 48,47 and 40 from the AC, GA and ATG libraries, respectively. Polymerase chain reaction (PCR) primer pairs for 99 of these inserts were selected using DesignerPcR version 1.03 (Research Genetics) and synthesized (Integrated DNA Technologies) for further evaluation. All forward primers were tailed on their $5^{\prime}$ end with the

Correspondence: C.D. Nelson, Fax: 228-832-0130;

E-mail: dananelson@fs.fed.us
M13forward(-29) sequence, CACGACGTTGTAAAACGAC, allowing fluorescent dye (6-FAM, VIC, NED or PET, Applied Biosystems) incorporation in PCR when using a dye-labelled M13forward(-29) primer. All reverse primers were tailed on their 5' end with a PIG tail, GTTTCTT (Brownstein et al. 1996), forcing nontemplated dA additions to the amplified fragments.

Synthesized primer pairs were screened for polymorphism against 23 T. caroliniana samples collected from seven typical hemlock sites distributed across the mountainous area of North Carolina and adjacent South Carolina. In addition, a subset of 63 markers that cleanly amplified in T. caroliniana was evaluated for use in six additional Tsuga species: eastern hemlock (T. canadensis), $n=31$; Chinese hemlock (T. chinensis), $n=5$; mountain hemlock (T. mertensiana), $n=3$; northern Japanese hemlock (T. diversifolia), $n=3$; southern Japanese hemlock (T. sieboldii), $n=2$; and western hemlock (T. heterophylla), $n=4$. All DNA samples were isolated from fresh leaf samples using DNeasy 96 Plant Kit (QIAGEN). PCR and allele separation were performed as previously described (Josserand et al. 2006), with the exception that a hot-start monoclonal antibody (GeneCraft, $\mathrm{GmbH}$ ) was used in the PCR. An internal LIZ 600 size standard was run with each sample and alleles were sized using the local southern algorithm and binned with GeneMapper 3.7 software (Applied Biosystems). Analyses of genotype frequencies, genotypic disequilibrium and exact tests for $P$ values were conducted with GenePop 3.4 (http:// genepop.curtin.edu.au/). 
Of the 99 primer pairs tested with T. caroliniana samples, 34 amplified consistent allelic profiles and of these, 31 were polymorphic (Table 1). Among the polymorphic loci, the number of alleles per locus ranged from two to seven (average $=3.4$ ), with observed and expected heterozygosities ranging from 0.043 to 1.0 (average $=0.208$ ) and from 0.043 to 0.768 (average $=0.475$ ), respectively. Tests of HardyWeinberg equilibrium found that 23 loci were not in equi-

Table 1 Results for 31 microsatellite loci isolated from Tsuga caroliniana and tested on a sample of 23 T. caroliniana trees

\begin{tabular}{|c|c|c|c|c|c|c|c|c|c|}
\hline $\begin{array}{l}\text { Locus } \\
\text { name }\end{array}$ & $\begin{array}{l}\text { Source } \\
\text { library }\end{array}$ & $\begin{array}{l}\text { GenBank } \\
\text { Accession }\end{array}$ & $\begin{array}{l}\text { Observed } \\
\text { repeat motif }\end{array}$ & Primer sequences $\left(5^{\prime}-3^{\prime}\right)$ & $\begin{array}{l}\text { Number of } \\
\text { alleles: range } \\
\text { in size (bp) }\end{array}$ & $H_{\mathrm{O}}$ & $H_{\mathrm{E}}$ & $\begin{array}{l}\text { HWE } \\
(P \\
\text { value })\end{array}$ & $\begin{array}{l}\text { Significant } \\
\text { LD with } \\
\text { TcSI locus }\end{array}$ \\
\hline TcSI_002 & $\mathrm{AC}$ & BV726470 & $(\mathrm{AC})_{23}$ & $\begin{array}{l}\text { F: GCATTAGGATAATCATTCCAGA } \\
\text { R: AGAGGGAAAAATAACCACTGAG }\end{array}$ & $2: 233-235$ & $0.043^{x s}$ & 0.122 & 0.002 & \\
\hline TcSI_004 & $\mathrm{AC}$ & BV726471 & $(\mathrm{AC})_{27}$ & $\begin{array}{l}\text { F: GCCAGATTTTGTCTAATCC } \\
\text { R: TTGGTGTCTTATCTAATCAGTG }\end{array}$ & 5: $270-283$ & $0.174 x \mathrm{~s}$ & 0.414 & 0.068 & 080 \\
\hline TcSI_006 & $\mathrm{AC}$ & BV726538 & $(\mathrm{GA})_{11}$ & $\begin{array}{l}\text { F: ATCCCCCAATTAGCCATG } \\
\text { R: AGGGCGGATCAATGTTTG }\end{array}$ & 3: 113-161 & 1.000 & 0.619 & $0.000^{*}$ & \\
\hline TcSI_007 & $\mathrm{AC}$ & BV726472 & $(\mathrm{CA})_{16}(\mathrm{AT})_{8}$ & $\begin{array}{l}\text { F: CAAGGCAACAACTATCTTTAGC } \\
\text { R: CAACTTTCTTCCAАCСTCTGT }\end{array}$ & $2: 261-265$ & 1.000 & 0.500 & $0.000^{*}$ & \\
\hline TcSI_008 & $\mathrm{AC}$ & BV726473 & $(\mathrm{AC})_{16}(\mathrm{AT})_{9}$ & $\begin{array}{l}\text { F: ACGGCTTTTCATTTGATTG } \\
\text { R: CATTCGGTAACATTTGATGC }\end{array}$ & 3: 119-155 & 1.000 & 0.619 & $0.000^{*}$ & \\
\hline TcSI_009 & GA & BV726474 & $(\mathrm{TC})_{30}$ & $\begin{array}{l}\text { F: TGGTTACAAGGTTCTGACAGTC } \\
\text { R: CTACCCAATGCTAAGGTGATT }\end{array}$ & $6: 250-272$ & $0.087 \mathrm{xs}$ & 0.438 & $0.000^{*}$ & \\
\hline TcSI_012 & $\mathrm{GA}$ & BV726476 & $(\mathrm{TC})_{12}$ & $\begin{array}{l}\text { F: CCATTTGCTAGACATGCAATAC } \\
\text { R: TTTCAGATGGACAGACGTAATG }\end{array}$ & 2: 249-251 & 0.087 & 0.159 & 0.030 & \\
\hline TcSI_014 & $\mathrm{GA}$ & BV726478 & $(\mathrm{TC})_{18}(\mathrm{CA})_{8}$ & $\begin{array}{l}\text { F: CCAGATGGAATGGTAGGTG } \\
\text { R: AATGGGTGAGAGATAATGTGAG }\end{array}$ & 2: 167-169 & $0.130^{x s}$ & 0.364 & 0.002 & 040,076 \\
\hline TcSI_022 & $\mathrm{AC}$ & BV726483 & $(\mathrm{AC})_{19}$ & $\begin{array}{l}\text { F: CCAAATTCAGAATGTCATCC } \\
\text { R: TTGAAGGAATAGTGGTTGATG }\end{array}$ & 3: $221-225$ & $0.136^{\mathrm{xs}}$ & 0.501 & $0.000^{*}$ & \\
\hline TcSI_027 & $\mathrm{AC}$ & BV726487 & $(\mathrm{TG})_{15}$ & $\begin{array}{l}\text { F: AAATCCGTCAAGTGTGCTAC } \\
\text { R: CTCCAGTCACCATAАACСTTC }\end{array}$ & 3: 180-184 & $0.043^{\mathrm{xs}}$ & 0.417 & $0.000^{*}$ & 078 \\
\hline TcSI_030 & $A C$ & BV726489 & $(\mathrm{AC})_{13}$ & $\begin{array}{l}\text { F: GCTGCATACTGTTTACCCTAAA } \\
\text { R: TAGGCTTGTGTTCCTCTGC }\end{array}$ & 2: 201-203 & $0.043^{x s}$ & 0.405 & $0.000^{*}$ & \\
\hline TcSI_032 & $\mathrm{AC}$ & BV726490 & $(\mathrm{AT})_{10}(\mathrm{TG})_{6}(\mathrm{TG})_{9}$ & $\begin{array}{l}\text { F: TACGGACCATCGGCTATG } \\
\text { R: ATGATTATGCCCCGTCAAT }\end{array}$ & 2: 185-187 & $0.217 x s$ & 0.405 & 0.026 & \\
\hline TcSI_040 & GA & BV726493 & $(\mathrm{AG})_{27}$ & $\begin{array}{l}\text { F: CAATGCCCAAAGAACAAGTC } \\
\text { R: GGAGGAAGGACACGAAGAAG }\end{array}$ & 5: 113-123 & $0.087 x \mathrm{xs}$ & 0.740 & $0.000^{*}$ & \\
\hline TcSI_044 & $\mathrm{GA}$ & BV726495 & $(\mathrm{TC})_{7}(\mathrm{CT})_{7}$ & $\begin{array}{l}\text { F: TCTTCTCTTCTACTGCCTCTAA } \\
\text { R: AGGATAAGTCGTGTTTCTGAG }\end{array}$ & 5: 150-158 & $0.087 \mathrm{xs}$ & 0.400 & $0.000^{*}$ & \\
\hline TcSI_055 & $\mathrm{AC}$ & BV726502 & $(\mathrm{CA})_{20}(\mathrm{AT})_{6}$ & $\begin{array}{l}\text { F: TTCCTCAACCCATTATACAATC } \\
\text { R: TCAAGGATGAACCATTAGAATC }\end{array}$ & 2: 268-270 & $0.087 x s$ & 0.386 & $0.000^{*}$ & \\
\hline TcSI_057 & $\mathrm{AC}$ & BV726504 & $(\mathrm{AC})_{13}$ & $\begin{array}{l}\text { F: TAACCCATCCCTTTACAAAAG } \\
\text { R: TAGCCGTGTGGTCATAGTTAG }\end{array}$ & 2: 135-137 & $0.130 \mathrm{xs}$ & 0.405 & $0.001^{*}$ & $\begin{array}{l}066,075 \\
079\end{array}$ \\
\hline TcSI_060 & $\mathrm{AC}$ & BV726507 & $(\mathrm{AC})_{20}$ & $\begin{array}{l}\text { F: CAACAATCATGTCACTCCATA } \\
\text { R: GTCGTCGGATTTTGTCTC }\end{array}$ & 3: 99-103 & $0.087 \mathrm{xs}$ & 0.322 & $0.001^{*}$ & \\
\hline TcSI_061 & $A C$ & BV726508 & $(\mathrm{AC})_{24}$ & $\begin{array}{l}\text { F: TGTGCTTGGGAATTATCTTAAC } \\
\text { R: CTTCAAGACCCTGCCTAAAG }\end{array}$ & 2: 136-138 & 0.043 & 0.043 & 0.915 & \\
\hline TcSI_062 & $A C$ & BV726509 & $(\mathrm{AT})_{5}(\mathrm{TG})_{32}$ & $\begin{array}{l}\text { F: ACGCCAACAAACTTCCTTT } \\
\text { R: GAGGGGTATGGAGATGTCC }\end{array}$ & $6: 268-299$ & $0.136^{\mathrm{xs}}$ & 0.689 & $0.000^{*}$ & 066,080 \\
\hline TcSI_066 & $A C$ & BV726513 & $(\mathrm{TG})_{5}(\mathrm{GT})_{27}$ & $\begin{array}{l}\text { F: TGCACCATGCAATGAAAC } \\
\text { R: GCСCСАTCAАATGAАATG }\end{array}$ & $2: 217-218$ & $0.043^{x s}$ & 0.485 & $0.000^{*}$ & 075,080 \\
\hline TcSI_067 & $A C$ & BV726514 & $(\mathrm{AC})_{16}$ & $\begin{array}{l}\text { F: AATATGGGATGTGGTAACTGTG } \\
\text { R: CTCTGGTGTTTTTGTGTATGGT }\end{array}$ & 2: $242-244$ & $0.043 \times s$ & 0.315 & $0.000^{*}$ & \\
\hline TcSI_074 & $\mathrm{GA}$ & BV726519 & $(\mathrm{GA})_{17}$ & $\begin{array}{l}\text { F: CAAAGATTGGAGACGAACTTG } \\
\text { R: CCTTACAGAAATACCCCATCAC }\end{array}$ & 5: 169-179 & $0.217 \mathrm{xs}$ & 0.584 & 0.002 & 076 \\
\hline TcSI_075 & $\mathrm{GA}$ & BV726520 & $(\mathrm{GA})_{20}$ & $\begin{array}{l}\text { F: CTCGTTGAGCATGATTGTAAC } \\
\text { R: ATAGTTCCACTGCAACCTCATA }\end{array}$ & 3: 174-188 & $0.130 x s$ & 0.559 & $0.000^{*}$ & 080,089 \\
\hline TcSI_076 & $\mathrm{GA}$ & BV726521 & $(\mathrm{GA})_{30}$ & $\begin{array}{l}\text { F: AGAGCACACGAGAAATAGATTG } \\
\text { R: TATTCCAACCATAGGTCTCAAC }\end{array}$ & 7: 158-176 & $0.217 \mathrm{xs}$ & 0.768 & $0.000^{*}$ & \\
\hline TcSI_078 & GA & BV726522 & $(\mathrm{CT})_{20}$ & $\begin{array}{l}\text { F: AACCCATACAAAGGATGCC } \\
\text { R: GAGAGGTAGGAAGGGATGTTG }\end{array}$ & 5: $235-243$ & $0.217 x \mathrm{~s}$ & 0.719 & $0.000^{*}$ & \\
\hline TcSI_079 & GA & BV726523 & $(\mathrm{AG})_{16}$ & $\begin{array}{l}\text { F: CCСААССААССТTTTTGTAА } \\
\text { R: AGCACАСТСТСАСАСТTСАСТС }\end{array}$ & 3: 290-294 & $0.130^{x s}$ & 0.523 & $0.000^{*}$ & 089 \\
\hline
\end{tabular}


Table 1 Continued

\begin{tabular}{|c|c|c|c|c|c|c|c|c|c|}
\hline $\begin{array}{l}\text { Locus } \\
\text { name }\end{array}$ & $\begin{array}{l}\text { Source } \\
\text { library }\end{array}$ & $\begin{array}{l}\text { GenBank } \\
\text { Accession }\end{array}$ & $\begin{array}{l}\text { Observed } \\
\text { repeat motif }\end{array}$ & Primer sequences $\left(5^{\prime}-3^{\prime}\right)$ & $\begin{array}{l}\text { Number of } \\
\text { alleles: range } \\
\text { in size (bp) }\end{array}$ & $H_{\mathrm{O}}$ & $H_{\mathrm{E}}$ & $\begin{array}{l}\text { HWE } \\
(P \\
\text { value })\end{array}$ & $\begin{array}{l}\text { Significant } \\
\text { LD with } \\
\text { TcSI locus }\end{array}$ \\
\hline TcSI_080 & GA & BV726524 & $(\mathrm{CT})_{25}$ & $\begin{array}{l}\text { F: TCCTCCTCATTTTCACACTCAC } \\
\text { R: CCAAATCCAACATTCCCTAGTT }\end{array}$ & 4: $224-230$ & $0.087 x \mathrm{~s}$ & 0.717 & $0.000^{*}$ & 087 \\
\hline TcSI_083 & GA & BV726527 & $(\mathrm{AG})_{25}$ & $\begin{array}{l}\text { F: GTGTCCTGAGGTGTGTTGTC } \\
\text { R: TTGGATGTTTACGATGATGG }\end{array}$ & 3: 234-239 & 1.000 & 0.572 & $0.000^{*}$ & \\
\hline TcSI_085 & GA & BV726528 & $(\mathrm{TC})_{20}$ & $\begin{array}{l}\text { F: TCTTCCCTTCTCCTCATAGGTC } \\
\text { R: GATTTTCTGTGCATGTGTGTTC }\end{array}$ & $3: 280-284$ & $0.174 \times s$ & 0.436 & 0.014 & \\
\hline TcSI_087 & GA & BV726530 & $(\mathrm{GA})_{23}$ & $\begin{array}{l}\text { F: TTTGTTCTTGGAACTTGACATC } \\
\text { R: CTTTTATGCAGCAAATTCACTC }\end{array}$ & 4: 279-285 & $0.217 \mathrm{xs}$ & 0.606 & $0.001^{*}$ & \\
\hline TcSI_089 & GA & BV726532 & $(\mathrm{TC})_{19}$ & $\begin{array}{l}\text { F: GGGAAATGTGCCTTAGTTTAA } \\
\text { R: GGAAAGGAGAAAAACACACAG }\end{array}$ & 3: $115-134$ & $0.130 \times s$ & 0.517 & $0.000^{*}$ & \\
\hline
\end{tabular}

Repeat structure and primer sequences and observed number of alleles, allele size range (base pairs, bp), heterozygosity values (observed, $H_{\mathrm{O}}$ and expected, $H_{\mathrm{E}}$ ), and $P$ values for exact tests of Hardy-Weinberg equilibrium (HWE), and TcSI locus number exhibiting significant $(P<0.05)$ genotypic disequilibrium in paired tests $(\mathrm{LD})$. Loci with $H_{\mathrm{O}}$ values marked with $\mathrm{xs}$ had an excess of homozygotes, as determined by Micro-Checker analysis (van Oosterhout et al. 2004). $P$ values marked with * are significant following sequential Bonferroni correction for $P<0.05$ (Holm 1979).

Table 2 Marker transferability results for 16 Tsuga caroliniana-derived markers tested with six Tsuga species of various sample sizes ( $n$ ), observed number of alleles and allele size range in base pairs

\begin{tabular}{|c|c|c|c|c|c|c|}
\hline Locus & $\begin{array}{l}\text { T. canadensis } \\
n=31\end{array}$ & $\begin{array}{l}\text { T. chinensis } \\
n=5\end{array}$ & $\begin{array}{l}\text { T. mertensiana } \\
n=3\end{array}$ & $\begin{array}{l}\text { T. diversifolia } \\
n=3\end{array}$ & $\begin{array}{l}\text { T. sieboldii } \\
n=2\end{array}$ & $\begin{array}{l}\text { T. heterophylla } \\
n=4\end{array}$ \\
\hline TcSI_004 & - & 6: 235-291 & 1: 304 & 2: $230-338$ & 2: $254-316$ & 3: 226-232 \\
\hline TcSI_008 & - & - & 3: 103-115 & $1: 146$ & - & 6: 106-144 \\
\hline TCSI_012 & 11: 243-268 & 6: $230-259$ & 2: $239-241$ & 2: 209-239 & 1: 241 & 3: 239-247 \\
\hline TcSI_014 & - & 5: 164-195 & - & - & - & - \\
\hline TcSI_029 & 3: 119-125 & 5: 105-123 & 2: 109-121 & 2: 103-105 & 1: 108 & 3: 107-113 \\
\hline TcSI_030 & - & - & 1: 201 & - & - & - \\
\hline TcSI_040 & 10: 91-121 & $2: 80-85$ & - & 2: 129-131 & 1: 117 & 6: 95-115 \\
\hline TcSI_052 & 3: $207-230$ & 1: 219 & 1: 225 & $1: 218$ & 1: 222 & - \\
\hline TcSI_060 & - & 4: $113-129$ & 1: 109 & - & 2: $111-124$ & 1: 109 \\
\hline TcSI_061 & - & - & - & - & - & 2: 104-108 \\
\hline TcSI_067 & - & - & - & - & - & 3: 245-252 \\
\hline TcSI_071 & - & - & 2: 112-114 & - & - & - \\
\hline TcSI_076 & - & 6: 126-192 & - & - & 2: 149-182 & - \\
\hline TcSI_080 & 6: 216-232 & 5: 209-234 & - & - & - & $6: 221-239$ \\
\hline TcSI_083 & 8: 247-265 & - & - & - & - & - \\
\hline TcSI_089 & - & $6: 117-142$ & - & 2: $134-140$ & 1: 128 & - \\
\hline
\end{tabular}

-, no amplification or difficult to interpret amplification products. The following loci (GenBank Accession) were monomorphic in $T$. caroliniana: TcSI_029 (BV726488), TcSI_052 (BV726500) and TcSI_071 (BV726517).

librium $(P<0.05$, Table 1$)$. Tests for two-locus genotypic disequilibrium found 16 significant locus pairs out of 435 tests after sequential Bonferroni correction for $P<0.05$ (Holm 1979). Tests for null alleles, using the Dunn-Sidak correction for Monte Carlo simulations in the program Micro-Checker (van Oosterhout et al. 2004), found 25 loci that have an excess of homozygotes (Table 1). Their mean estimated proportion of null alleles across all null estimators was 0.35 with a $95 \%$ confidence interval of \pm 0.03 , indicating little variation in the proportion of excess homozygosity among the loci. The results of these three tests together are consistent with inbreeding within geographically isolated stands (Farjon 1990) and do not support the presence of widespread linkage disequilibrium or high null allele frequencies.

Microsatellite primers developed from some conifer species can be amplified in related taxa (Echt et al. 1999; Chagné et al. 2004; Josserand et al.2006). Of the 34 primer pairs that cleanly amplified in T. caroliniana, 16 (47\%) amplified in the other six species (Table 2), with the greatest proportion being transferable to T. chinensis (29\%, 10 markers) and 
the least to $T$. canadensis $(18 \%, 6$ markers). Of the three markers that were monomorphic for T. caroliniana, TcSI_029, TcSI_052 and TcSI_071, each successfully amplified polymorphic loci in at least one other Tsuga species. TcSI_029 appeared polymorphic in five other species and TcSI_052 and TcSI_071 appeared polymorphic in one other species each (Table 2).

Clearly the microsatellite markers described in this paper will enhance population genetic studies of Carolina hemlock and other hemlock species, as well as assist interand intraspecies breeding programmes (Bentz et al. 2002; Pooler et al. 2002) working to develop HWA resistant populations for species conservation and restoration.

\section{Acknowledgements}

We thank the Southern Research Station, John Frampton and Bill Dvorak of North Carolina State University for their support of hemlock genetics research through Cooperative Agreement (SRS 05-CA-11330126-210) and Jim Vose (US Forest Service, Coweta Hydrologic Laboratory). In addition, we thank Dennis Deemer (Southern Institute of Forest Genetics) for technical assistance and the following for providing Tsuga spp. samples: Susan Bentz and Richard Olsen, National Arboretum, Washington, DC; Dan Moeller, Hoyt Arboretum, Portland, OR; Barbara Crane, US Forest Service, Southern Region, Atlanta, GA; Valerie Hipkins, US Forest Service, National Forest Genetics Laboratory, Placerville, CA; and the staffs of Camcore and the JC Raulston Arboretum, North Carolina State University, Raleigh, NC.

\section{References}

Bentz SE, Riedel LGH, Pooler MR, Townsend AM (2002) Hybridization and self-compatibility in controlled pollinations of eastern North American and Asian hemlock (Tsuga) species. Journal of Arboriculture, 28, 200-205.

Brownstein MJ, Carpten JD, Smith JR (1996) Modulation of nontemplated nucleotide addition by Taq polymerase: primer modifications that facilitate genotyping. BioTechniques, 20, 1004-1010.

Chagné D, Chaumeil P, Ramboer A et al. (2004) Cross-species transferability and mapping of genomic and cDNA SSRs in pines. Theoretical and Applied Genetics, 109, 1204-1214.

Echt CS, Vendramin GG, Nelson CD, Marquardt P (1999) Microsatellite DNA as shared genetic markers among conifer species. Canadian Journal of Forest Research, 29, 365-371.

Farjon A (1990) Pinaceae: Drawings and Descriptions of the Genera Abies, Cedrus, Pseudolarix, Keteleeria, Nothotsuga, Tsuga, Cathaya, Pseudostuga, Larix and Picea. Koeltz Scientific Books, Konigstein, Germany.

Holm S (1979) A simple sequentially rejective multiple test procedure. Scandinavian Journal of Statistics, 6, 65-70.

Josserand SA, Potter KM, Johnson G, Bowen A, Frampton J, Nelson CD (2006) Isolation and characterization of microsatellite markers in Fraser fir (Abies fraseri). Molecular Ecology Notes, 6, 65-68.

McClure MS, Salom SM, Shields KS (2003) Hemlock Woolly Adelgid. FHTET-2001-03. Forest Health Technology Enterprise Team, US Department of Agriculture, Forest Service, Morgantown, West Virginia.

Pooler MR, Riedel LGH, Bentz SE, Townsend AM (2002) Molecular markers used to verify interspecific hybridization between hemlock (Tsuga) species. Journal of the American Society of Horticultural Science, 1227, 623-627.

van Oosterhout C, Hutchinson WF, Wills DPM, Shipley P (2004) Micro-Checker: software for identifying and correcting genotyping errors in microsatellite data. Molecular Ecology Notes, 4, 535-538. 